\title{
Tangence
}

\section{La dépouille d'un rêve}

\section{Georges Schlocker}

Numéro 46, décembre 1994

Un théâtre de passage

URI : https://id.erudit.org/iderudit/025838ar

DOI : https://doi.org/10.7202/025838ar

Aller au sommaire du numéro

Éditeur(s)

Tangence

ISSN

0226-9554 (imprimé)

1710-0305 (numérique)

Découvrir la revue

Citer cet article

Schlocker, G. (1994). La dépouille d'un rêve. Tangence, (46), 29-37.

https://doi.org/10.7202/025838ar d'utilisation que vous pouvez consulter en ligne.

https://apropos.erudit.org/fr/usagers/politique-dutilisation/ 


\section{La dépouille d'un rêve \\ Georges Schlocker}

À côté du rythme et de l'édifice de la parole, il y a au thêâtre un rythme et un édifice du mouvement, des mouvements, qui doit laisser dans l'esprit la mémoire d'un tout complet...

Antonin Artaud, Projet de lettre à Jean Paulban

On ne se trompe pas en situant la naissance du théâtre d'images au Festival de Nancy de l'année 1971. Après les sept heures de représentation de Regard du sourd de Robert Wilson, le public se sentait le témoin d'un événement historique. En effet, la finalité du message théâtral venait de prendre une direction jusqu'alors insoupçonnée. La tradition rhétoricienne du théâtre français, que même les esthétiques les plus contradictoires (classiques, romantiques, sociologiques, voire politiques) avaient à peine égratignée, se retrouvait inopérante et mise au rancart puisque le verbe était pratiquement banni de la scène. Ce que les personnages désiraient exprimer se traduisait par l'agencement des corps des acteurs, autrement dit par une chorégraphie muette, et par un assemblage dans l'espace de couleurs, de costumes et de gestes collectifs. Les moyens expressifs devenaient le message en soi : la volonté de transformer la scène en composition picturale s'exprimait à travers une construction spectaculaire savamment réglée. L'auteur de cet ensorcellement, Robert Wilson, expliquait dix-sept ans plus tard, en mai 1988, lors d'un colloque au Théâtre dramatique royal de Stockholm: "Je n'ai pas fait d'études de théâtre, mon background, c'est l'architecture et les arts plastiques." Et plus loin dans la même communication:

Je suis toujours troublé par le fait qu'en général au théâtre, ce que nous voyons est toujours une illustration ou une ornementation de ce que nous entendons. Nous envisageons le théâtre comme s'il était un texte ou un audio-book.

Le point de départ de son travail scénique était un visual book, autrement dit un répertoire de tableaux dont chacun articulait un 
30

message dépassant largement sa signification première. Un parallèle s'imposait alors à l'esprit avec les performances figées des tableaux "vivants" du peintre-sculpteur Kienholz. Mais il est vrai qu'entre Wilson et Kienholz, il y avait une différence de taille: le premier créait un monde féerique où des éléments concrets de la réalité quotidienne décollaient du réel pour s'épanouir dans un univers imaginaire, alors que le second s'acharnait à faire la critique réaliste de la société américaine à travers la représentation immobile de ces éléments concrets. La dynamique intrinsèque du contenu différait aussi considérablement. Ainsi Kienholz exprimait dans ses construction théâtrales une violence sur le point d'exploser, alors que Wilson faisait défiler dans les tableaux vivants de ses débuts, j'entends ceux qu'il a faits en France dans les années soixante-dix (comme A Letter for Queen Victoria), un univers imprégné de suavité, sorte de règne ininterrompu d'Alice au pays des merveilles.

$\mathrm{Au}$ début, la douceur de l'inspiration wilsonnienne (qui découle de l'intention pédagogique de l'établissement de sourdsmuets, la Byrd Hofmann School, qui avait commandé le spectacle) fut un élément constitutif de ce langage scénique. En effet, il s'agissait non seulement d'un défilé d'images dont les liens faisaient peu de cas de la logique, mais aussi d'un cortège d'images déclenchant dans l'âme du spectateur une "plus-value" signifiante. Les figures bien délimitées en actions sur la scène en appelaient à l'«infigurable ", bien au-delà des apparitions offertes au regard.

Dans la première décennie de son existence, ce type de théâtre explorait avec un entrain irrépressible les moyens d'expression plastiques en mouvement. Un autre Américain, Richard Foreman, arriva alors à Paris, avec son Ontological-Hysteric Theatre dont les images sur scène, loin du statisme wilsonnien, traduisaient l'agressivité de la société américaine. Suivant ses déclarations, il était venu à Paris pour trouver hors d'Amérique un autre retentissement aux tortures de son inconscient. En 1973, il introduisit, avec sa pièce Classical Therapy or a Week under the Influence, une problématique contestataire dont la symbolique très personnelle des images, dotée d'un indubitable relent surréaliste, fascinait en tant que document d'une subjectivité exacerbée, et signalait d'ores et déjà le danger inhérent à la communication par images, à savoir l'arbitraire de son invention. Loin de la rationnalisation verbale, ces configurations plastiques se basaient principalement sur la névrose de leur créateur et sur l'attraction des 
oppositions subies par son esprit à même leur charge affective. De spectacle en spectacle, cette dernière devenait plus importante: puisqu'une blessure ressentie à la naissance du créateur aiguillonnait ce travail scénique, son travail était voué à devenir un lieu de souffrance soit immédiate, soit cryptée par le symbolisme des images. Dans ce cas, la rapidité des déplacements sur scène, le choc des corps qui s'y affrontaient, les cris ou la musique assourdissants signalaient un état exceptionnel considéré comme l'état affectif "normal" sur scène. Dans une longue explication des raisons pour lesquelles il avait quitté New York pour travailler à Paris pendant dix ans, Foreman définissait en 1989 les deux pôles de son inspiration artistique:

On n'a pas compris comment une "non-pensée" organisée de manière rythmique essayait de transformer et de transcender un thème d'une importance capitale - et cela afin de montrer que l'équilibre individuel était possible au milieu des circonstances les plus éprouvantes et les plus désespérées. ${ }^{1}$

Un parallèle avec un phénomène des beaux-arts s'impose ici. À la fin des années soixante-dix, une exposition mondiale d'art plastique qui se tient tous les quatre ans à Kassel proposait le thème de la "mythologie personnelle" pour caractériser des ouvres faisant fi de toute "lisibilité objective". Elles traduisent, selon l'explication des organisateurs-historiens de l'art, un message dépouillé de toute référence universellement vérifiable. L'art témoigne d'un système de symboles et de formes (l'un souvent synonyme de l'autre) qui naissent suivant les impulsions d'une subjectivité qui découvre une certaine cohérence dans le seul acte de la transmission. L'œuvre est susceptible de provoquer la "catharsis", au sens aristotélicien, dans la mesure où elle témoigne d'une clarification douloureuse ou extatique de l'âme de l'artiste. Ainsi, par l'image théâtrale, Foreman va-t-il au-devant d'une discussion de sa "non-pensée" avec le way of life qu'il veut fuir.

L'image thêâtrale se voit ainsi chargée d'une signification existentielle. Elle n'est que vaguement déchiffrable par le spectateur, mais le rébus que lui met sous les yeux l'action scénique lui inspire une angoisse réelle. Il y sent une volonté de crypter le message associé à un désir de se faire comprendre. Dans le texte

1 Tbéâtre/publique, no 86, p. 40. 
cité plus haut, Foreman explique ce va-et-vient entre compréhension et incompréhension:

J'ai toujours visé, non pas un sentiment du moi, mais un sentiment de l'existence. Le sentiment du moi, les gens l'obtiennent, à mon avis, en s'exprimant et en trouvant dans l'autre la certitude rassurante que ce qu'ils disent est compris et accepté. Mais cela ne leur donne pas un sentiment d'existence réelle: l'autre, par son consentement, ne fait que les rejoindre dans un mensonge socialement conditionné. Le sentiment de l'existence s'obtient peut-être en abandonnant toute idée de communication, en se laissant guider par certains élans difficiles à définir. C'est cela le moteur du travail que j'ai accompli à Paris.

"Oh images, vous suffisez à mon bonheur", disait un personnage de Classical Therapy. Les metteurs en scène à travers le monde répétaient cette profession de foi. Il est vrai que les images représentant la dépouille d'un rêve ont eu un essor foudroyant. Il suffit de penser au théâtre dansé, un genre issu justement du cortège des images sur scène. Pina Bausch est devenue célèbre dans le monde entier en traduisant en pas dansés certaines scènes de son autobiographie. En effet, dans ses premières chorégraphies, son expérience passée assure le lien avec l'onirisme des scènes. Gestes et mouvements adressent un message qui dépasse à chaque fois la forme de l'expression choisie. Le contenu s'affirme par le dépassement du geste. Ainsi, l'agencement des images garantit la thêâtralité des tableaux. Or, comme on sait, la théâtralité exerce de nos jours une fascination extraordinaire non seulement dans le domaine de l'art, mais aussi dans la vie réelle. Lorsque la réalité est placée sur plusieurs niveaux et parait non pas univoque, mais plurivoque, la simulation n'est point entachée de mensonge. Aragon a créé le terme du "mentir-vrai "qui résume bien la réponse de notre époque à ce phénomène esthétique. En effet, plus la médiatisation est appréciée, plus la scène, livrée aux images, flatte la prédilection pour le fauxsemblant, parangon du rythme furtif des formes.

L'image pourtant ne veut pas tromper le spectateur. Elle lui fait parfaitement comprendre qu'elle est image, qu'elle n'est que provisoire. Chez Robert Wilson tout comme chez ses émules, la lenteur de l'évolution des images est irrésistible. Ce thêâtre ne tend pas à exprimer une marche douce et lente, il évoque avant tout la progression qui ne s'arrête jamais. Celle-ci répond certes à 
une volonté théâtrale exhibitionniste, mais sa technique, qui consiste à faire naître une image d'une autre, fait aussi image, référence au fondu enchaîné filmique.

Sous cet angle, une remarque de Richard Foreman mérite notre attention. En effet, le mélange des images lles plus disparates a été un procédé exploité par les surréalistes. Mais pour son théâtre, il postule une autre tradition: celle de Gertrude Stein. Celle-ci, dont le Faust a été porté à la scène et par Robert Wilson et par Richard Foreman, aimait le télescopage des images dans la fiction:

Gertrude Stein se saisissait d'une image, l'effaçait pour en faire surgir une autre et ainsi de suite. Cet incessant effacement des images permet de se faire une idée du paysage qui les a "générées". ${ }^{2}$

L'irrationalité et la dynamique dégagée par une telle cascade d'images pourrait déboucher sur l'appréhension du "tout complet " auquel fait allusion Artaud. Elle fait entrevoir une réalité "supérieure" derrière la matérialité des composants de l'image. Les apparences changeantes produites sur la scène poussent l'observateur vers le plan du sens situé hors d'atteinte du contrôle rationnel. Ce dernier ne produirait que des visions étriquées, bêtement logiques.

Depuis quelques années, le Centre dramatique d'Osona produit, sous l'impulsion créatrice de Wladislaw Znorko, des spectacles image et son appelés Cosmos Kolej. À Gennevilliers, on a pu assister à plusieurs de ces spectacles, notamment Cbveik au terminus du monde (1992) et Ulysse à l'envers (1994). Voici des histoires imaginaires situées au seuil de la Première Guerre mondiale, évoquant dans un cadre aussi délabré que fantaisiste un catalogue de déréliction des êtres dont l'humour n'est point absent. Ces productions s'attachent à des personnages imaginaires comme Chveik ou Ulysse, mais ne négligent point leur contenu historique. La définition qu'en donne son auteur fait appel à un terme fort caractéristique de ce style de théâtre. Il explique:

Depuis sa création, le Cosmos Kolej est à la quête d'un langage thêâtral universel. Ses spectacles, appareillages plastiques et

2 Théâtre/publique, $\mathrm{n}^{\circ} 36$, novembre-décembre 1988, p. 8 
34

poétiques, opèrent, sans scrupules, les distorsions du temps et du récit en dévoilant au grand jour les ferrailleurs et les mécaniciens qui fourbissent la chaudière de nos songes.

Stylistiquement, les créations de ce théâtre sont tributaires du théâtre de foire. Ce théâtre table sur un double dépaysement créé, primo, par l'effet de foire avec ses marginaux et ses paumés, domaine maintes fois visité depuis le début du siècle, exprimant le regret romantique devant le paradis perdu, secundo, par l'évocation de l'Europe centrale, depuis toujours réservoir inépuisable de la nostalgie collective latine. Historiquement, le spectacle dirige ses lumières (plus souvent ses pénombres) sur les victimes, les laissés-pour-compte: l'univers matériellement déglingué de ceux que la politique ou la société ont recrachés.

Notons que, sur le public français, les reflets d'une civilisation d'un rêve pur et jamais possédé agissent puissamment. Le théâtre de Joseph Nadi, un émule du théâtre dansé, en apporte un autre témoignage. La Hongrie de la double monarchie y refleurit; son romantisme champêtre mi-souriant mi-grotesque évoque un contexte bien désuet aujourd'hui. Les tentatives du Théâtre du Radeau, situé au Mans, sont du même ordre. François Tanguy en appelle, dans la plupart de ses productions, à un monde frustre et délabré de quelque contrée imaginaire du centre de l'Europe sur lequel le soleil paraît ne jamais se lever. Chez lui aussi, le phénomène de la citation joue un rôle prépondérant. Ainsi le Radeau a-t-il consacré une production à Faust et une autre aux fragments de texte récemment découverts de Woyzeck. Dans un décor champêtre, une maison de ferme ouvre ses battants et laisse entrevoir des espaces dans lesquels flottent des brouillards et des murmures de menaces indéfinissables. Les textes sont débités par bribes, comme s'il s'agissait d'un écho lointain des images. Le point zéro de la conscience et le bas de l'échelle sociale sont ainsi ébauchés en même temps, ainsi par cette synesthésie: "l'œeil écoutait", pour reprendre la formule de Claudel.

Mais comment passer en revue ce théâtre produit surtout en France et omettre le véritable créateur de l'image stridente, qui n'est point français, à savoir Tadeusz Kantor? Son Teatr Cricot 2, à Cracovie, puise à différentes esthétiques, à l'image même de la personnalité complexe de son créateur. Le surréalisme, avec son aspect merveilleux, inspire le peintre Kantor; la technique de la Performance, avec son intention de créer le réel immédiatement devant le spectateur, l'a entraîné sur le chemin de la découverte 
de son propre art. Un des traits de son originalité consiste dans la recherche d'un temps révolu qu'il essaie d'infuser dans la réalité du moment, mais toujours en préservant ce qu'il appelle son "étrangeté". Les images chez lui oscillent entre le proche et le lointain. Elles ont à chaque fois le poids et le pathos du passé, d'événements de l'histoire que la scène ressuscite. Voici comment, en 1974, il décrit le phénomène de l'étrangeté, émanation du rêve compris non comme une fuite, mais comme un rappel d'être disparus loin de toute psychologie:

Je désire atteindre un degré d'étrangeté tel que le public puisse le percevoir douloureusement. On peut y parvenir à travers une situation capable de créer la barrière voulue... Une autre possibilité existe qui naît dans nos rêves. Endormis, nous rencontrons des personnages qui nous furent très proches et qui brusquement, on ne sait pourquoi, se comportent comme s'ils ne nous avaient jamais connus, comme s'ils étaient devenus des ÉTRANGERS. ${ }^{3}$

La classe morte était le premier spécimen de résurrection théâtrale qui repoussait les personnages dans une situation d'étrangers. Les différentes scènes étaient comparables à des aphorismes spatiaux ancrés dans un contexte historique précis. Nous n'assistions donc pas à un ballet d'inventions cocasses et arbitraires, mais bien à une remémoration exacte de ce qui fut. Le texte de la scène appelée Toast nous éclaire parfaitement:

Une valse banale, sentimentale: "Si une fois encore le bon temps revenait ", grâce aux répétitions infinies, se transforme en un appel vain et désespéré...

Par cette répétition insistante, insupportable, qui agit comme l'hypnose, elle parvient presque à ressusciter ce temps passé, perdu et mort. Sur scène, l'espace d'un moment, nous voilà témoins de ce miracle évident... ${ }^{4}$

Une des constantes du théâtre de l'image est la construction d'une œuvre fermée qui accuse le caractère d'une construction intellectuelle. Le fait que les images s'emboitent les unes dans les autres ou se talonnent au rythme de leur apparition sur scène ne change rien à leur caractère bien enfermé dans les éléments et les effets de ce langage unique, enchâssé une fois pour toutes à l'intérieur de son cadre. La puissance du théâtre de Kantor

3 Les voies de la création thêâtrale, vol. XI, Paris, Éditions du CNRS, 1983, p. $79-80$.

$4 \quad$ Ibid., p. 91. 
consiste, au contraire, dans le caractère d'une œuvre jamais étanche à l'histoire de la collectivité et aux émotions qu'elle provoque au fil des ans. Est-ce une contradiction quand il déclare en 1987, en présentant La classe morte à Paris: "L'art doit être énigmatique. Fermé jusqu'à l'inaccessible."

Or cette énigme n'a pas ses racines dans l'ahistoricité ni dans l'ésotérisme. Elle est affaire de langage, c'est-à-dire qu'elle crée des raccourcis communs à l'auditoire. Si ce message reste gratuit et se résume dans un jeu d'effets qui ne fait qu'épater son public, s'il s'avère insipide face aux grandes interrogations de l'époque, alors il apparaît comme un exercice formel sans prise sur la réalité. Plus encore, il dévoile sa caducité.

À l'heure actuelle, où les grands modèles stylistiques (esthétiques) se sont épanouis depuis longtemps, la scène charrie à profusion et sans inspiration nouvelle une inflation d'images tantôt violentes, tantôt sentimentales. Ainsi s'établit un procédé au fonctionnement quasi mécanique que même des artistes conscients de leur art arrivent difficilement à combattre. C'est le cas d'Achim Freyer, un homme de théâtre allemand fort respectable. Lui aussi sait manier à la perfection des éléments picturaux qui ensorcellent. En utilisant ce langage, il reste conscient de l'exigence de parler de sujets importants pour l'homme, donc de ne pas s'adonner à de la pure virtuosité magique. Il recourt dans ses mises en scène à une volonté de transcender la signification. Hélas, le langage en tant que tel n'apparaît que répétitif; la prouesse, et non la révélation, reste son lot.

Qui plus est: le récit en images devient une expression artistique à la mode, correspond donc à une attente du public. Il y a des textes qui sont d'entrée de jeu destinés à l'expression corporelle. Peter Handke en a écrit un: L'beure où nous ne savions rien l'un de l'autre. Il propose des êtres entièrement enfermés dans une sorte d'autisme; aucune faille ne les rendrait perméable à autrui. À la Schaubühne de Berlin, dans la mise en scène de Luc Bondy, ces personnages marchent dans un décor merveilleusement rehaussé par des zones de lumière et d'ombre. Un monde d'une sensibilité parfaite nous est suggéré. Seulement, tout cela ne représente qu'un écho, le retentissement d'un retentissement; c'est de l'artisanat artistique appliqué avec grande classe. On pourrait aussi bien y voir un produit de consommation pour public avancé ou se prenant pour tel. 
L'absence de rigueur analytique derrière les images produit des stéréotypes: le théâtre des images est sans conteste à la mode. Lorsque, dans Enfonçures, Didier-Georges Gabily veut faire allusion à la "vraie vie" qui hante le poète (en l'occurrence Hölderlin, autre citation à la mode), les images évoquées sur scène font toutes référence à une civilisation agreste reculée. L'image peut donc traquer l'invisible derrière le visible, dévoiler le tragique de l'histoire ou tout simplement révéler la "nonpensée". Voilà sa vertu tout comme sa faiblesse. 